\title{
Desigualdades sociais do impacto das quedas de idosos na qualidade de vida relacionada à saúde
}

\author{
Social inequalities in the impact of falls on health-related quality \\ of life among older adults
}

Mariana Mapelli de Paiva (https://orcid.org/0000-0003-4947-7523) ${ }^{1}$

Margareth Guimarães Lima (https://orcid.org/0000-0001-6996-0745) ${ }^{1}$

Marilisa Berti de Azevedo Barros (https://orcid.org/0000-0003-3974-195X) ${ }^{1}$
${ }^{1}$ Departamento de Saúde Coletiva, Faculdade de Ciências Médicas, Universidade Estadual de Campinas. R. Tessália Vieira de Camargo 126, Cidade Universitária Zeferino Vaz. 13083-887 Campinas SP Brasil.marianamapelli@ hotmail.com

\begin{abstract}
The aim of the present study was to determine the association between falls and health-related quality of life (HRQoL) among older adults considering different demographic and socioeconomic characteristics. This study was developed with data from the Household Health Survey conducted in the city of Campinas, Brazil, in 2014 and 2015. HRQoL was investigated using the eight domains and two components of the SF-36 questionnaire. Simple and multiple linear regression analyses were performed with the aid of the Stata 15.0 program to determine the association between falls and HRQoL according to sex, age, income and schooling. Significant declines in the scores of the physical functioning, role physical and bodily pain domains as well as the physical component were found among women (not men) and individuals with a lower income. Among individuals aged 75 years or older and those with less schooling, declines occurred in these same domains as well as in the role emotional and mental health domains. The results reveal that the impact of falls on HRQoL differs depending on the socioeconomic and demographic characteristics of older adults, indicating that specific care strategies should target more vulnerable subgroups, with attention given to emotional aspects.
\end{abstract}

Key words Quality of life, Accident by falls, Elder health
Resumo Oobjetivo deste artigo é verificar a associação entre queda e qualidade de vida relacionada à saúde considerando idosos de diferentes segmentos demográficos e socioeconômicos. O estudo foi desenvolvido com dados do Inquérito Domiciliar de Saúde (ISACamp) realizado em Campinas, SP, em 2014 e 2015. A QVRS foi avaliada por meio dos oito domínios e dos dois componentes do SF-36. Utilizou-se o Stata 15.0 para as análises de regressão linear simples e múltipla a fim de determinar as associações entre quedas e QVRS segundo sexo, idade, renda e escolaridade. Declínios significativos dos escores em capacidade funcional, aspectos físicos e dor, e no componente físico foram observados nos idosos do sexo feminino (não no masculino) e naqueles de menor renda; nos indivíduos com 75 anos ou mais e menor escolaridade, além destas dimensões, também ocorreram declínios nos aspectos emocionais e na saúde mental. Os resultados revelam que o impacto das quedas na QVRS difere conforme o segmento socioeconômico e demográfico do idoso, indicando que cuidados e estratégias especiais devem ser dedicados aos subgrupos mais vulneráveis, incluindo a atenção aos aspectos emocionais.

Palavras-chaves Qualidade de vida, Acidente por quedas, Saúde do idoso 


\section{Introdução}

Segundo dados do Instituto Brasileiro de Geografia e Estatística (IBGE), de 2004 a 2015 o contingente da população idosa no país passou de $9,8 \%$ para $14,3 \%$, e as projeções indicam que em 2070 o número de idosos representará 35\% da população total ${ }^{1}$.

Com o rápido envelhecimento populacional são cada vez mais presentes as discussões relacionadas aos problemas de saúde e aos fatores que ocasionam impactos na qualidade de vida dos idosos $^{1,2}$. Neste contexto, um dos problemas de saúde relevantes na atualidade são as quedas que são muito mais frequentes neste subgrupo etário da população. Entre os idosos, a literatura aponta que esses acidentes são mais prevalentes no sexo feminino, naqueles com idade mais avançada, nos que apresentam maior número de morbidades e que utilizam maior número de medicamentos ${ }^{3,4}$. As quedas, ocasionando lesões mais ou menos graves, mesmo que não coloquem a vida do idoso em risco e que não deixem sequelas, podem comprometer de forma importante o estado de saúde ${ }^{5,6}$ e a qualidade de vida dos afetados ${ }^{7}$.

O processo de envelhecimento, com os declínios biológicos da senescência, implica em aumento de vulnerabilidades de natureza biológica que interagem com contextos socioeconômicos e psicossociais ${ }^{8}$ acelerando ou retardando esse processo. Nesta perspectiva, considerando-se a influência da posição socioeconômica e das condições de vida na adoção de hábitos saudáveis, na prevenção de doenças e no acesso a conhecimentos e a cuidados à saúde ${ }^{9}$, investigações sobre problemas específicos, como as quedas e seus impactos na QVRS, precisariam considerar a sua ocorrência em diferentes estratos socioeconômicos.

É conhecido que a desigualdade social se expressa em diversas dimensões e eventos da saúde. Em relação à prevalência de quedas, as pesquisas não têm detectado diferenças de prevalências entre estratos socioeconômicos ${ }^{10,11}$, mas também é importante avaliar se o impacto das quedas acomete de maneira desigual a população situada em diferentes posições sociais e neste sentido as pesquisas são escassas. A associação das quedas de idosos com QVRS considerando a posição socioeconômica foi desenvolvida por um estudo brasileiro $^{12}$, que avaliou as diferenças segundo escolaridade. Apenas três pesquisas avaliaram o impacto das quedas na QVRS em relação a sexo ${ }^{12-}$ ${ }^{14}$ encontrando resultados divergentes. Enquanto Chang et al. ${ }^{13}$, em estudo desenvolvido em Taipei,
Taiwan, verificaram que as quedas produziram maiores declínios nos escores dos componentes físico e mental do SF-36 nas mulheres em comparação aos homens, em dois estudos brasileiros as quedas mostraram associação com alguns domínios do SF-36 em homens e não nas mulheres $^{12,14}$. Em relação a grupos etários os resultados dos poucos estudos realizados ${ }^{12-14}$ também diferem, com dois deles revelando maiores declínios associados a quedas nos idosos com idades mais avançadas $^{12,13}$.

Considerando o rápido aumento da população idosa, a maior vulnerabilidade para ocorrência de quedas deste segmento populacional e os prejuízos que as quedas acarretam na QVRS, além da escassez de pesquisas de base populacional acerca desta temática, esta pesquisa tem por objetivo verificar a associação entre queda e qualidade de vida relacionada à saúde, considerando idosos de diferentes segmentos demográficos e socioeconômicos.

\section{Métodos}

Trata-se de um estudo transversal, de base populacional, realizado com idosos não institucionalizados, residentes na área urbana do município de Campinas, São Paulo. Os dados do presente estudo são provenientes do inquérito domiciliar de saúde (ISACamp), realizado em 2014 e 2015. O ISACamp teve por objetivo obter informações sobre morbidades, comportamentos de saúde e uso de serviços de saúde relativas a três segmentos etários: adolescentes, adultos e idosos.

Para a seleção dos sujeitos utilizou-se amostragem probabilística, estratificada, por conglomerados e em dois estágios. No primeiro estágio, foram sorteados 70 setores censitários. No segundo estágio, foram selecionados os domicílios por meio de sorteio sistemático aplicado às relações atualizadas de domicílios existentes em cada um dos 70 setores censitários sorteados. O número de pessoas para compor a amostra foi obtido considerando-se a situação correspondente à máxima variabilidade para a frequência dos eventos estudados $(P=0,50)$, coeficiente de confiança de $95 \%$ na determinação dos intervalos de confiança $(z$ $=1,96)$, erro de amostragem entre 4 e 5 pontos percentuais e efeito de delineamento igual a 2, totalizando 1.000 adolescentes (10 a 19 anos), 1.400 adultos (20 a 59 anos) e 1.000 idosos (60 anos e mais). Para alcançar esse tamanho de amostra em cada domínio de idade, foram selecionados de forma independente 3.119, 1.029, 3.157 domi- 
cílios para entrevistas com adolescentes, adultos e idosos respectivamente, já considerando o percentual de perdas esperado.

As informações foram obtidas por meio de questionário estruturado, organizado em doze blocos temáticos, e aplicado em entrevistas domiciliares, realizadas com o uso de tablets por entrevistadores treinados e supervisionados. No caso de idosos com incapacidade de responder ao questionário foi solicitada ajuda de um familiar ou cuidador para efetuar a coleta das informações.

As variáveis dependentes analisadas foram as oito escalas do The Medical Outcomes Study 36Item Short-Form Health Survey (SF-36), versão 2, que mede o estado de saúde autoavaliado e é composto por 36 itens distribuídos em oito escalas: capacidade funcional, aspectos físicos, dor, estado geral de saúde, vitalidade, aspectos emocionais, aspectos sociais e saúde mental. As escalas podem ser sintetizadas em dois componentes (físico e mental). Este instrumento passou pelo processo de tradução e validação no Brasil ${ }^{15,16}$.

Para obter os escores do SF-36, de acordo com a metodologia proposta pelo instrumento, foram atribuídas pontuações a cada questão, conforme a resposta do entrevistado. Os escores obtidos em cada domínio foram convertidos para uma escala de zero a 100 , sendo que zero corresponde ao pior estado de saúde e 100 ao melhor ${ }^{17}$. Os componentes foram padronizados com os escores médios da população de Campinas, usando uma transformação linear com uma média de 50 e desvio padrão de 10 , recomendados pelo manual do instrumento ${ }^{17}$.

Utilizou-se como variável independente principal a ocorrência de quedas relatada por meio da pergunta: "O(a) $\mathrm{Sr}(\mathrm{a})$. sofreu alguma queda, seja leve ou grave, nos últimos 12 meses?”.

As variáveis utilizadas para estratificação foram: Sexo (masculino e feminino); Faixa etária (60 e 74 anos; 75 anos ou mais); Escolaridade em anos de estudo ( 0 a 4 anos; 5 anos e mais); Renda familiar mensal per capita em salários mínimos (de até 2,5 salários mínimos; $\geq 2,5$ salários mínimos).

Para ajuste das análises, além de sexo e idade, foi utilizada também a variável número de doenças crônicas (DC). Foi calculado o número de doenças crônicas reportadas pelos entrevistados, como diagnosticadas por médico ou outro profissional de saúde, e que constavam no cheklist: hipertensão arterial, diabetes, angina, infarto do miocárdio, tumor/câncer, artrite/reumatismo/ artrose, osteoporose, asma/bronquite/enfisema, rinite, sinusite, tendinite/lesão por esforço repe- titivo, varizes de membros inferiores, acidente vascular cerebral, colesterol elevado, doença de coluna/problema de coluna.

Foram estimadas as médias, erro padrão e intervalos de confiança de $95 \%$ dos escores de cada uma das oito escalas e para cada componente do SF-36. A associação entre QVRS e quedas foi verificada com o uso de regressão linear simples e múltipla, estimando os betacoeficientes, com ajustes pelas variáveis sexo, faixa etária e número de doenças crônicas. As análises foram realizadas com estratificação para sexo, idade, escolaridade e renda.

Todas as análises foram realizadas por meio do software Stata 15.0 (StataCorp, College Station, EUA) utilizando os comandos svy que incorporam as ponderações necessárias em decorrência do desenho amostral complexo.

O projeto do ISACamp 2014/2015 foi aprovado pelo Comitê de Ética em Pesquisa da Universidade Estadual de Campinas. O presente estudo também foi aprovado pelo CEP via Plataforma Brasil.

\section{Resultados}

Entre os domicílios que haviam sido sorteados para obter a amostra de idosos, as perdas totalizaram $6,8 \%$. Dos 1.168 idosos identificados nos domicílios sorteados, houve $14 \%$ de recusas e $1,5 \%$ de outras perdas, por não encontrar o morador em mais de três visitas. A população do estudo foi assim constituída por 986 idosos.

A prevalência de quedas no ano anterior a pesquisa foi de $17,1 \%$ (IC 95\%: 14,76-19,81). A população caracterizou-se por maior percentual de mulheres $(57,5 \%)$, de indivíduos entre 60-74 anos $(71,2 \%)$, com escolaridade de até quatro anos de estudo (57,7\%) e com renda mensal menor que 2,5 salários mínimos (71,2\%).

Considerando-se os resultados das análises ajustadas por sexo e/ou idade e número de doenças crónicas, verificou-se que em relação a sexo, apenas as mulheres apresentaram prejuízos, sendo estes encontrados nos escores das escalas de capacidade funcional, aspectos físicos, dor e no componente físico. Nos homens que sofreram quedas, não foi observado declínio significativo em nenhuma das escalas do SF-36 (Tabela 1).

Em relação à faixa etária, nos idosos com idades entre 60-74 anos, não houve diferença entre as médias dos escores do SF-36 segundo a ocorrência de quedas. Entre os idosos com 75 anos e mais verificou-se menores médias em cinco do- 
Tabela 1. Médias e erro padrão dos escores do SF-36 e betacoeficientes segundo ocorrência de quedas, por sexo. ISACamp 2014/2015, Campinas, São Paulo, Brasil.

\begin{tabular}{|c|c|c|c|}
\hline \multicolumn{4}{|c|}{ Masculino $(n=387)$} \\
\hline \multirow{2}{*}{ Domínios do SF-36 } & \multirow{2}{*}{$\begin{array}{c}\begin{array}{c}\text { Não sofreu quedas } \\
(n=339)\end{array} \\
\text { Médias (Erro padrão) }\end{array}$} & \multicolumn{2}{|c|}{ Sofreu quedas $(n=47)$} \\
\hline & & $\beta($ valor de p) & $\beta(\text { valor de } p)^{\star}$ \\
\hline Capacidade funcional & $77,1(1,6)$ & $-6,6(0,205)$ & $-2,9(0,470)$ \\
\hline Aspectos Físicos & $78,4(1,9)$ & $-5,4(0,349)$ & $-3,4(0,489)$ \\
\hline Dor & $77,3(1,6)$ & $-3,5(0,381)$ & $-2,9(0,376)$ \\
\hline Saúde Geral & $74,9(1,3)$ & $-6,3(0,005)$ & $-4,1(0,070)$ \\
\hline Vitalidade & $76,3(1,6)$ & $-0,9(0,799)$ & $1,2(0,649)$ \\
\hline Aspectos Emocionais & $87,1(1,5)$ & $-6,3(0,241)$ & $-5,6(0,207)$ \\
\hline Aspectos Sociais & $88,1(1,5)$ & $-1,0(0,772)$ & $2,4(0,467)$ \\
\hline Saúde Mental & $80,5(1,1)$ & $-5,6(0,091)$ & $-3,9(0,206)$ \\
\hline Componente Físico & $46,5(0,5)$ & $-2,7(0,123)$ & $-1,6(0,231)$ \\
\hline Componente Mental & $52,1(0,6)$ & $-1,8(0,308)$ & $-1,1(0,501)$ \\
\hline \multicolumn{4}{|c|}{ Feminino $(n=599)$} \\
\hline \multirow[t]{2}{*}{ Domínios do SF-36 } & $\begin{array}{c}\text { Não sofreu quedas } \\
(n=471)\end{array}$ & \multicolumn{2}{|c|}{ Sofreu quedas $(n=127)$} \\
\hline & Médias (Erro padrão) & $\beta($ valor de p) & $\beta(\text { valor de } p)^{\star}$ \\
\hline Capacidade funcional & $68,0(1,9)$ & $-12,4(0,002)$ & $-8,3(0,006)$ \\
\hline Aspectos Físicos & $74,4(1,8)$ & $-11,3(0,003)$ & $-8,1(0,013)$ \\
\hline Dor & $70,2(1,7)$ & $-14,2(0,000)$ & $-10,2(0,002)$ \\
\hline Saúde Geral & $72,2(1,4)$ & $-2,1(0,388)$ & $0,5(0,830)$ \\
\hline Vitalidade & $70,2(1,5)$ & $-5,7(0,063)$ & $-3,2(0,305)$ \\
\hline Aspectos Emocionais & $81,2(1,6)$ & $-6,4(0,049)$ & $-4,2(0,176)$ \\
\hline Aspectos Sociais & $81,4(1,2)$ & $-3,4(0,272)$ & $-1,8(0,556)$ \\
\hline Saúde Mental & $73,9(1,3)$ & $-3,6(0,119)$ & $-1,5(0,567)$ \\
\hline Componente Físico & $43,9(0,7)$ & $-5,2(0,001)$ & $-3,5(0,004)$ \\
\hline Componente Mental & $49,2(0,6)$ & $-0,4(0,781)$ & $0,09(0,949)$ \\
\hline
\end{tabular}

${ }^{*}$ Ajustado por faixa etária e número de doenças crônicas.

mínios do SF-36 (capacidade funcional, aspectos físicos, dor, aspectos emocionais, saúde mental) e no componente físico (Tabela 2).

Quando analisado o impacto das quedas na QVRS segundo o nível de escolaridade, verificouse que os idosos situados no segmento de menor nível (até quatro anos), apresentaram prejuízos nas escalas de capacidade funcional, aspectos físicos, dor, aspectos emocionais, saúde mental e no componente físico (Tabela 3). Não foram observadas associações nos idosos com maior escolaridade.

$\mathrm{Na}$ Tabela 4 observa-se que nos idosos do estrato de menor renda, as associações entre quedas e QVRS foram evidenciadas nas escalas de capacidade funcional, aspectos físicos, dor e no componente físico, sendo que nenhuma associação foi verificada no segmento de maior renda.

\section{Discussão}

O estudo permitiu mensurar os declínios dos escores de QVRS associados à ocorrência de quedas em idosos de diferentes estratos sociodemográficos. Idosos que sofreram quedas e que eram do sexo masculino, que tinham idade inferior a 75 anos, que pertenciam aos segmentos de maior escolaridade e de maior renda não apresentaram declínios significativos dos escores nos domínios do SF-36, em comparação aos que não haviam sofrido quedas. Idosos que sofreram quedas e que eram do sexo feminino e aqueles com menor renda apresentaram médias significativamente menores de QVRS, em comparação aos que não sofreram quedas, nos domínios de capacidade funcional, aspectos físicos e dor e no componente físico. Idosos com 75 anos ou mais e os de menor escolaridade, além destes domínios, também 
Tabela 2. Médias e erro padrão dos escores do SF-36 e betacoeficientes segundo ocorrência de quedas, por faixa etária. ISACamp 2014/2015, Campinas, São Paulo, Brasil.

\begin{tabular}{|c|c|c|c|}
\hline \multicolumn{4}{|c|}{$60-74$ anos $(n=664)$} \\
\hline \multirow[t]{2}{*}{ Domínios do SF-36 } & $\begin{array}{c}\text { Não sofreu quedas } \\
(n=561)\end{array}$ & \multicolumn{2}{|c|}{ Sofreu quedas $(n=103)$} \\
\hline & Médias (Erro padrão) & $\beta$ (valor de p) & $\boldsymbol{\beta}(\text { valor de } \mathbf{p})^{*}$ \\
\hline Capacidade funcional & $77,6(1,3)$ & $-8,1(0,006)$ & $-4,0(0,177)$ \\
\hline Aspectos Físicos & $79,4(1,5)$ & $-5,0(0,179)$ & $-2,0(0,617)$ \\
\hline Dor & $72,1(1,4)$ & $-10,8(0,003)$ & $-4,2(0,215)$ \\
\hline Saúde Geral & $73,8(1,2)$ & $-2,6(0,257)$ & $1,4(0,562)$ \\
\hline Vitalidade & $73,8(1,4)$ & $-3,6(0,226)$ & $0,6(0,847)$ \\
\hline Aspectos Emocionais & $85,3(1,2)$ & $-3,4(0,279)$ & $-0,4(0,902)$ \\
\hline Aspectos Sociais & $85,3(1,1)$ & $-2,1(0,460)$ & $2,1(0,480)$ \\
\hline Saúde Mental & $76,1(1,1)$ & $-3,5(0,152)$ & $0,5(0,858)$ \\
\hline Componente Físico & $46,2(0,5)$ & $-3,5(0,006)$ & $-1,6(0,182)$ \\
\hline Componente Mental & $50,0(0,6)$ & $-0,5(0,704)$ & $1,0(0,503)$ \\
\hline \multicolumn{4}{|c|}{75 anos e mais $(n=322)$} \\
\hline \multirow[t]{2}{*}{ Domínios do SF-36 } & $\begin{array}{c}\text { Não sofreu quedas } \\
(n=249)\end{array}$ & \multicolumn{2}{|c|}{ Sofreu quedas $(n=71)$} \\
\hline & Médias (Erro padrão) & $\beta$ (valor de p) & $\boldsymbol{\beta}(\text { valor de } \mathbf{p})^{*}$ \\
\hline Capacidade funcional & $57,5(2,2)$ & $-13,2(0,007)$ & $-9,3(0,023)$ \\
\hline Aspectos Físicos & $67,6(2,8)$ & $-15,8(0,002)$ & $-12,3(0,010)$ \\
\hline Dor & $76,9(1,8)$ & $-15,8(0,001)$ & $-14,1(0,002)$ \\
\hline Saúde Geral & $72,4(1,7)$ & $-5,4(0,055)$ & $-3,9(0,225)$ \\
\hline Vitalidade & $70,8(2,0)$ & $-7,4(0,024)$ & $-4,8(0,159)$ \\
\hline Aspectos Emocionais & $79,8(2,7)$ & $-12,4(0,013)$ & $-10,6(0,039)$ \\
\hline Aspectos Sociais & $81,8(2,1)$ & $-5,6(0,153)$ & $-4,1(0,321)$ \\
\hline Saúde Mental & $79,0(1,3)$ & $-9,0(0,001)$ & $-7,5(0,005)$ \\
\hline Componente Físico & $42,0(0,7)$ & $-6,0(0,002)$ & $-4,5(0,005)$ \\
\hline Componente Mental & $51,8(0,8)$ & $-2,9(0,106)$ & $-2,6(0,157)$ \\
\hline
\end{tabular}

${ }^{*}$ Ajustado por sexo e número de doenças crônicas.

tiveram declínios significativos nos domínios de aspectos emocionais e de saúde mental.

A associação da queda com prejuízo da QVRS detectada nesta pesquisa apenas no sexo feminino e não no masculino encontra-se em consonância com os resultados de estudo desenvolvido em Taiwan com 4.056 idosos que constatou que as quedas se associavam a maiores prejuízos nas mulheres em comparação aos homens, avaliando os componentes físico e mental do SF- $36^{13}$. Esses achados, entretanto, diferem dos observados em dois estudos brasileiros que detectaram declínios dos escores de domínios do SF-36 apenas em homens $^{12,14}$, sendo que em um deles ${ }^{12}$ a queda era relativa ao principal acidente ocorrido nos 12 meses anteriores à entrevista. As diferenças entre os resultados dos estudos podem decorrer das características (idades, morbidades, vulnerabilidades) das populações estudadas, dos instrumen- tos utilizados para coleta de informações sobre quedas e das variáveis consideradas para ajustes nos procedimentos de análise, entre outros. São necessários mais estudos para que estes aspectos possam ser melhor entendidos. O maior impacto das quedas na QVRS nas idosas poderia estar relacionado à maior perda de massa magra e de força muscular nas mulheres em comparação aos homens ${ }^{18}$.

A observação de declínio dos escores em cinco escalas e no componente físico do SF-36 apenas nos idosos com 75 anos ou mais, sem reduções significativas nos idosos mais jovens, concorda com o achado do estudo de Chang et al. ${ }^{13}$ que verificaram prejuízos crescentes com a idade no componente físico do SF-36 associados a quedas de idosos. As pesquisas apontam que a prevalência de quedas aumenta com a idade ${ }^{4,19}$, sendo então importante detectar se existem diferenças 
Tabela 3. Médias e erro padrão dos escores do SF-36 e betacoeficientes segundo ocorrência de quedas, por escolaridade. ISACamp 2014/2015, Campinas, São Paulo, Brasil.

\begin{tabular}{|c|c|c|c|}
\hline \multicolumn{4}{|c|}{0 a 4 anos $(n=604)$} \\
\hline \multirow{2}{*}{ Domínios do SF-36 } & \multirow{2}{*}{$\begin{array}{l}\text { Não sofreu quedas } \\
(\mathrm{n}=492)\end{array}$} & \multicolumn{2}{|c|}{ Sofreu quedas $(n=116)$} \\
\hline & & $\boldsymbol{\beta}$ (valor de p) & $\boldsymbol{\beta}(\text { valor de } \mathbf{p})^{\star}$ \\
\hline Capacidade funcional & $66,34(1,5)$ & $-13,4(0,000)$ & $-6,6(0,020)$ \\
\hline Aspectos Físicos & $72,1(1,8)$ & $-14,5(0,001)$ & $-9,8(0,010)$ \\
\hline Dor & $71,6(1,6)$ & $-13,9(0,000)$ & $-11,3(0,001)$ \\
\hline Saúde Geral & $71,1(1,3)$ & $-5,9(0,002)$ & $-3,0(0,138)$ \\
\hline Vitalidade & $71,5(1,3)$ & $-7,5(0,024)$ & $-4,4(0,124)$ \\
\hline Aspectos Emocionais & $80,7(1,4)$ & $-12,3(0,004)$ & $-9,2(0,019)$ \\
\hline Aspectos Sociais & $80,8(1,5)$ & $-5,9(0,109)$ & $-3,2(0,347)$ \\
\hline Saúde Mental & $75,7(1,2)$ & $-7,5(0,004)$ & $-5,6(0,023)$ \\
\hline Componente Físico & $43,3(0,5)$ & $-5,5(0,000)$ & $-3,3(0,003)$ \\
\hline Componente Mental & $50,0(0,6)$ & $-3,0(0,062)$ & $-2,6(0,099)$ \\
\hline \multicolumn{4}{|c|}{5 e mais $(n=376)$} \\
\hline \multirow[t]{2}{*}{ Domínios do SF-36 } & $\begin{array}{l}\text { Não sofreu quedas } \\
\qquad(n=318)\end{array}$ & \multicolumn{2}{|c|}{ Sofreu quedas $(n=58)$} \\
\hline & Médias (Erro padrão) & $\beta($ valor de p) & $\beta(\text { valor de } p)^{*}$ \\
\hline Capacidade funcional & $79,9(1,9)$ & $-8,6(0,037)$ & $-3,6(0,341)$ \\
\hline Aspectos Físicos & $81,6(1,8)$ & $-1,8(0,648)$ & $2,3(0,582)$ \\
\hline Dor & $75,6(1,4)$ & $-8,6(0,021)$ & $-3,1(0,397)$ \\
\hline Saúde Geral & $76,5(1,3)$ & $0,2(0,942)$ & $3,4(0,168)$ \\
\hline Vitalidade & $74,9(1,7)$ & $-1,2(0,649)$ & $3,3(0,282)$ \\
\hline Aspectos Emocionais & $87,9(1,6)$ & $2,0(0,539)$ & $4,4(0,222)$ \\
\hline Aspectos Sociais & $89,1(1,4)$ & $1,0(0,733)$ & $4,0(0,204)$ \\
\hline Saúde Mental & $78,4(1,3)$ & $-1,1(0,623)$ & $1,7(0,576)$ \\
\hline Componente Físico & $47,4(0,6)$ & $-3,4(0,018)$ & $-1,3(0,316)$ \\
\hline Componente Mental & $51,1(0,7)$ & $1,7(0,200)$ & $2,6(0,099)$ \\
\hline
\end{tabular}

${ }^{\star}$ Ajustado por sexo, faixa etária e número de doenças crônicas.

do impacto das quedas na QVRS considerando as diferentes faixas de idade dos idosos. No presente estudo maior número de domínios mostraramse afetados nos idosos de 75 anos ou mais que sofreram queda de que o observado em estudo prévio realizado na mesma localidade ${ }^{12}$.

O maior impacto das quedas na QVRS com o avanço da idade está relacionado ao processo de progressivas alterações estruturais e funcionais, dentre elas diminuição de massa, força e função muscular, perdas na estabilidade e dinâmica articular e alterações sensoriais, dentre outras. Tais alterações afetam os mecanismos de marcha e equilíbrio aumentando o risco de quedas ${ }^{20-22} \mathrm{e}$, também favorecem que as consequências das quedas sejam mais graves ${ }^{17}$.

Quanto ao nível de escolaridade, os achados desta pesquisa identificaram prejuízos associados a quedas na QVRS apenas nos idosos com até quatro anos de escolaridade. O único estudo que avaliou a influência da escolaridade na associação de quedas com QVRS também detectou prejuízos na QV apenas entre os menos escolarizados, mas apenas nas escalas de aspectos físicos e sociais do SF-36 ${ }^{12}$. Entende-se que é importante investigar, na avaliação multidimensional do idoso, o nível de escolaridade, pois é um fator responsável por facilitar ou dificultar a compreensão das orientações em relação aos fatores responsáveis pelas quedas, as formas de prevenção e as implicações na QV. E neste segmento social é importante considerar os impactos das quedas na saúde mental e nos aspectos emocionais dos idosos que sofreram quedas.

Em relação à renda, verificou-se que os idosos com renda de até 2,5 salários mínimos, na presença de quedas, apresentam prejuízos nas escalas de capacidade funcional, aspectos físicos 
Tabela 4. Médias e erro padrão dos escores do SF-36 e betacoeficientes segundo ocorrência de quedas, por renda. ISACamp 2014/2015, Campinas, São Paulo, Brasil.

\begin{tabular}{|c|c|c|c|}
\hline \multicolumn{4}{|c|}{ Até 2,5 salários mínimos $(\mathrm{n}=749)$} \\
\hline \multirow{2}{*}{ Domínios do SF-36 } & $\begin{array}{l}\text { Não sofreu quedas } \\
\qquad(n=609)\end{array}$ & \multicolumn{2}{|c|}{ Sofreu quedas $(n=139)$} \\
\hline & Médias (Erro padrão) & $\beta($ valor de p) & $\beta(\text { valor de } p)^{*}$ \\
\hline Capacidade funcional & $70,3(1,7)$ & $-13,2(0,000)$ & $-7,2(0,004)$ \\
\hline Aspectos Físicos & $74,7(1,8)$ & $-11,3(0,002)$ & $-6,9(0,031)$ \\
\hline Dor & $71,3(1,4)$ & $-12,1(0,00)$ & $-8,4(0,006)$ \\
\hline Saúde Geral & $73,1(1,2)$ & $-5,5(0,007)$ & $-1,9(0,324)$ \\
\hline Vitalidade & $72,4(1,4)$ & $-7,8(0,005)$ & $-4,0(0,100)$ \\
\hline Aspectos Emocionais & $82,5(1,5)$ & $-8,9(0,006)$ & $-5,4(0,067)$ \\
\hline Aspectos Sociais & $82,7(1,3)$ & $-4,9(0,085)$ & $-1,0(0,690)$ \\
\hline Saúde Mental & $76,0(1,1)$ & $-6,6(0,002)$ & $-3,4(0,122)$ \\
\hline Componente Físico & $44,3(0,6)$ & $-5,0(0,000)$ & $-2,9(0,005)$ \\
\hline Componente Mental & $50,1(0,6)$ & $-2,1(0,070)$ & $-0,9(0,458)$ \\
\hline \multicolumn{4}{|c|}{$\geq 2,5$ Salários mínimos $(\mathrm{n}=237)$} \\
\hline \multirow[t]{2}{*}{ Domínios do SF-36 } & $\begin{array}{l}\text { Não sofreu quedas } \\
\qquad(n=201)\end{array}$ & \multicolumn{2}{|c|}{ Sofreu quedas $(n=35)$} \\
\hline & Médias (Erro padrão) & $\beta($ valor de p) & $\boldsymbol{\beta}(\text { valor de } \mathbf{p})^{\star}$ \\
\hline Capacidade funcional & $77,1(2,0)$ & $-7,8(0,207)$ & $-2,5(0,577)$ \\
\hline Aspectos Físicos & $80,2(1,9)$ & $-6,0(0,348)$ & $-1,9(0,732)$ \\
\hline Dor & $78,9(1,7)$ & $-11,1(0,046)$ & $-5,4(0,301)$ \\
\hline Saúde Geral & $74,3(1,7)$ & $1,4(0,712)$ & $4,3(0,228)$ \\
\hline Vitalidade & $74,5(2,1)$ & $2,9(0,382)$ & $6,5(0,061)$ \\
\hline Aspectos Emocionais & $87,3(1,7)$ & $-1,5(0,690)$ & $1,5(0,712)$ \\
\hline Aspectos Sociais & $88,9(1,6)$ & $0,7(0,840)$ & $3,0(0,458)$ \\
\hline Saúde Mental & $79,1(1,4)$ & $-0,5(0,863)$ & $1,0(0,751)$ \\
\hline Componente Físico & $47,1(0,6)$ & $-4,0(0,095)$ & $-1,8(0,267)$ \\
\hline Componente Mental & $51,5(0,7)$ & $1,6(0,397)$ & $2,1(0,260)$ \\
\hline
\end{tabular}

${ }^{*}$ Ajustado por sexo, faixa etária e número de doenças crônicas.

e dor e no componente físico. No segmento de melhor renda nenhum declínio foi estatisticamente significativo. Pesquisas envolvendo idosos têm identificado associações entre a prevalência de quedas e nível econômico ${ }^{3,21,23}$, porém não foram identificados estudos que tenham avaliado o impacto das quedas na QV segundo estratos de renda, revelando lacuna importante na literatura médica. A renda é um dos atributos que influenciam fortemente a saúde e a $\mathrm{QV}^{24,25}$.

Os achados em relação à associação entre quedas e QVRS, diferenciada segundo escolaridade e renda podem contribuir para ampliar o conhecimento sobre as consequências das quedas considerando o grau de vulnerabilidade social dos idosos ${ }^{26}$. As relações entre status socioeconômico e saúde são complexas e influenciadas por fatores de dimensões políticas, sociais e econômi- $\operatorname{cas}^{27}$. Aqueles que vivem em situações de vulnerabilidade social experimentando condições de privação material, com maior nível de estresse, menores opções de escolhas, maior probabilidade de comportamentos de riscos relacionados à saúde e redução de acesso aos serviços de saúde além de maior risco de quedas sofrem consequências mais intensas desses eventos ${ }^{28}$.

Destaque-se que a escala de dor foi a que apresentou o maior declínio nos idosos dos segmentos sociodemográficos afetados. As lesões provocadas pelas quedas podem ser leves, como escoriações e contusões, ou mais graves, como as fraturas ${ }^{5}$, e todas costumam resultar em dor e interferir na realização das atividades diárias. Ressalte-se que as quedas são responsáveis por lesões cujas múltiplas consequências, incluindo a dor, confrontam o idoso com sua fragilidade confi- 
gurando ameaça à sua segurança, autonomia e independência nas realizações das atividades diárias e sociais ${ }^{29}$.

Outras escalas prejudicadas na ocorrência de quedas foram os aspectos emocionais e a saúde mental entre idosos de 75 anos e mais e no segmento de menor escolaridade. O impacto das quedas na saúde mental relaciona-se às consequências que o evento provoca, como o medo de cair, que é preditivo de novas quedas e que pode levar à diminuição da confiança, da autonomia $\mathrm{e}$ da capacidade funcional, e aumento da percepção de fragilidade e de sentimentos negativos ${ }^{5,14}$.

Algumas limitações do estudo precisam ser mencionadas. Trata-se de um estudo transversal que não permite verificar relações causais entre as variáveis estudadas. É preciso considerar o viés de informação, uma vez que os dados foram obtidos por meio de entrevistas, e o possível viés de memória, pois as quedas foram referentes aos últimos 12 meses. Além disso, é preciso considerar que a qualidade de vida pode ser influenciada por diversos fatores além das quedas, embora as análises tenham sido ajustadas por variáveis demográficas (sexo e idade) e pelo número de doenças crônicas a fim de minimizar possíveis confundi-

\section{Colaboradores}

MM Paiva participou da análise e interpretação dos dados; e escrita e revisão crítica do manuscrito. MG Lima participou da interpretação dos dados e revisão crítica do manuscrito. MBA Barros participou da concepção e orientação do trabalho; interpretação dos dados e revisão crítica do manuscrito. mentos. Também é preciso considerar que foram analisados muitos desfechos e o nível de significância adotado foi $\mathrm{p}<0,05$. Observe-se, porém, que para a maior parte das associações encontradas, o valor de p foi menor ou igual a 0,01 . Em contrapartida, trata-se de um estudo com métodos padronizados e supervisionados de coleta de dados, com amostra representativa da população do município, que buscou estimular as lembranças dos idosos em relação às quedas leves e graves dos últimos 12 meses, e utilizou-se o SF-36, que é um instrumento amplamente utilizado e com estudo de validação feito no Brasil ${ }^{15}$.

$\mathrm{O}$ estudo traz informações novas sobre a associação das quedas com a qualidade de vida detectando a existência de desigualdades socioeconômicas no impacto das quedas, pois o grau de prejuízo depende do segmento social e demográfico a que o idoso pertence. O estudo contribui para o conhecimento acerca de uma temática pouco estudada e sugere que tais aspectos precisam ser mais pesquisados e considerados nas ações de políticas públicas voltadas à saúde dos idosos, com o intuito de reduzir a ocorrência das quedas e minimizar os impactos que provocam na qualidade de vida, principalmente nos segmentos sociodemográficos mais vulneráveis. 


\section{Referências}

1. Instituto Brasileiro de Geografia e Estatística (IBGE). Sintese de indicadores sociais: uma análise das condições de vida da população brasileira. Rio de Janeiro: IBGE; 2016.

2. Brasil. Ministério da Saúde (MS). Envelhecimento e Saúde da Pessoa Idosa. Brasília: MS; 2006.

3. Vieira LS, Gomes AP, Bierhals IO, Farías-Antúnez S, Ribeiro CG, Miranda VIA, Lutz BH, Barbosa-Silva TG, Lima NP, Bertoldi AD, Tomasi E. Falls among older adults in the South of Brazil: prevalence and determinants. Rev Saude Publica 2018; 52:22.

4. Nascimento JS, Tavares DMS. Prevalência e fatores associados a quedas em idosos. Texto Context Enferm 2016; 25(2):e0360015.

5. Barbosa KTF, Fernandes MGM, Oliveira FMRL, Santos KFO, Pereira MA. Queda em idosos: associação com morbidade e capacidade funcional. Rev Enferm UFPE Line 2013; 7(8):5068-5075.

6. Souza AQ, Pegorari MS, Nascimento JS, Oliveira PB, Tavares DMS. Incidência e fatores preditivos de quedas em idosos na comunidade: um estudo longitudinal. Cien Saude Colet 2019; 24(9):3507-3516.

7. Thiem U, Klaaben-Mielke R, Trampisch U, Moschny A, Pientka L, Hinrichs T. Falls and EQ-5D rated quality of life in community-dwelling seniors with concurrent chronic diseases: A cross-sectional study. Health Qual Life Outcomes 2014; 12:2.

8. Rodrigues NO, Neri AL. Vulnerabilidade social, individual e programática em idosos da comunidade: dados do estudo FIBRA, Campinas, SP, Brasil. Cien Saude Colet 2012; 17(8):2129-2139.

9. Ribeiro AP, Souza ER, Atie S, Souza AC, Schilithz AO. A influência das quedas na qualidade de vida de idosos. Cien Saude Colet 2008; 13(4):1265-1273.

10. Siqueira FV, Facchini LA, Silveira DS, Piccini RX, Tomasi E, Thumé E, Silva SM, Dilélio A. Prevalence of falls in elderly in Brazil: a countrywide analysis. Cad Saude Publica 2011; 27(9):1819-1826.

11. Perracini MR, Ramos LR. Fatores associados a quedas em uma coorte de idosos residentes na comunidade. Rev Saúde Pública 2002; 36(6):709-716.

12. Rodrigues IG, Lima MG, Barros MBA. Falls and health-related quality of life (SF-36) in elderly people-ISACAMP 2008. Health 2013; 5(12):49-57.

13. Chang NT, Chi LY, Yang NP, Chou P. The impact of falls and fear of falling on health-related quality of life in taiwanese elderly. J Community Health Nurs 2010; 27(2):84-95.

14. Pimentel WRT, Pagotto V, Nakatani AYK, Pereira LV, Menezes RL. Quedas e qualidade de vida: associação com aspectos emocionais em idosos comunitários. Geriatr Gerontol Aging 2015; 9(2):42-48.

15. Ciconelli RM, Ferraz MB, Santos W, Meinão I, Quaresma MR. Tradução para a língua portuguesa e validação do questionário genérico de avaliação de qualidade de vida SF-36 (Brasil SF-36). Rev Bras Reumatol 1999; 39:143-150.
16. Laguardia J, Campos MR, Travassos CM, Najar AL, Anjos LA, Vasconcellos MM. Psychometric evaluation of the SF-36 (v.2) questionnaire in a probability sample of Brazilian households: Results of the survey Pesquisa Dimensões Sociais das Desigualdades (PDSD), Brazil, 2008. Health Qual Life Outcomes 2011; 9(1):61.

17. Ware JE, Kosinski M, Bjorner JB, Turner-bowker DM, Gandek B, Maruish ME. User's manual for the SF-36v2 ${ }^{\circledR}$ Health Survey. $2^{\text {a }}$ ed. Lincoln: Quality Metric Incorporated; 2007.

18. Fried LP, Tangen CM, Walston J, Newman AB, Hirsch C, Gottdiener J. Frailty in older adults: Evidence for a phenotype. J Gerontol Med Sci 2001; 56(3):146-157.

19. Rodrigues IG, Fraga GP, Barros MBA. Quedas em idosos: fatores associados em estudo de base populacional. Rev Bras Epidemiol 2014; 17(9):705-718.

20. Cruz DT, Ribeiro LC, Vieira MT, Teixeira MTB, Bastos RR, Leite ICG. Prevalência de quedas e fatores associados em idosos. Rev Saude Publica 2012; 46(1):138146.

21. Bekibele CO, Gureje O. Fall incidence in a population of elderly persons in Nigeria. Gerontology 2010; 56(3):278-283.

22. Gill T, Taylor AW, Pengelly A. A population-based survey of factors relating to the prevalence of falls in older people. Gerontology 2005; 51(5):340-345.

23. Minayo MCS, Hartz ZMA, Buss PM. Qualidade de vida e saúde: um debate necessário. Cien Saude Colet 2000; 5(1):7-18.

24. Bortolotto CC, Mola CL, Tovo-Rodrigues L. Qualidade de vida em adultos de zona rural no Sul do Brasil : estudo de base populacional. Rev Saude Publica 2018; 52(Supl. 1):4s.

25. Abreu DROM, Azevedo RCS, Silva AMC, Reiners AAO, Abreu HCA. Fatores associados à recorrência de quedas em uma coorte de idosos. Cien Saude Colet 2016; 21(11):3439-3446.

26. World Health Organization (WHO). Closing the gap in a generation: Health equity through action the social determinants of health. Genebra: WHO; 2008.

27. World Health Organization (WHO). Preventing Chronic Diseases: a vital investment. Genebra: WHO; 2005.

28. Lilian K, Celich S, Galon C. Chronic pain in elderly and its influence in daily activities and social living. Rev Bras Geriatr Gerontol 2009; 12(3):345-359.

Artigo apresentado em 30/05/2019

Aprovado em 07/08/2019

Versão final apresentada em 22/11/2019 
